Historic, Archive Document

Do not assume content reflects current scientific knowledge, policies, or practices. 



$$
\begin{aligned}
& \text { B U L B B } \\
& \text { PR I C E } \\
& \text { L I S T }
\end{aligned}
$$

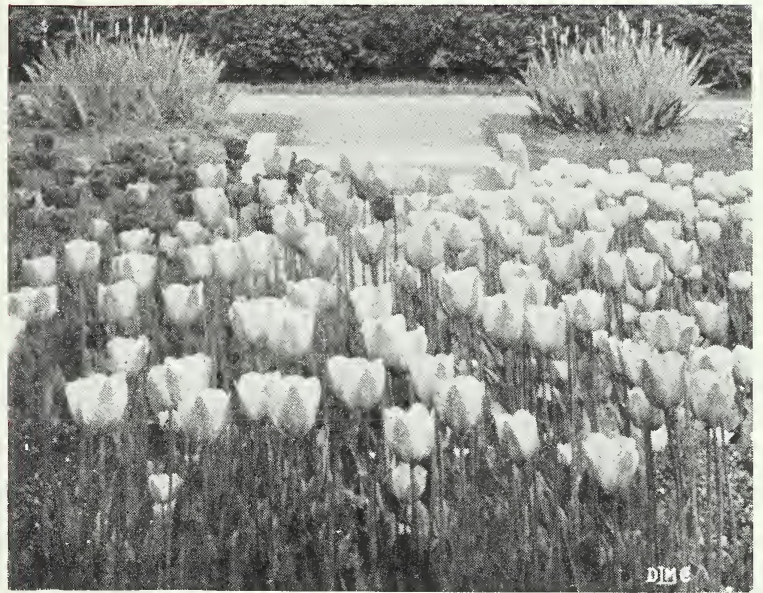

STANDARD

BULB CO. INC.

105 West 28th Street

New York, N. Y.

tr

Phone: LACkawanna 5790 


\section{LILY BULBS}

(Ready for immediate shipment)

LILIUM AURATUM

Per case

$8-9 \quad(180$ in a case $) \ldots \ldots \ldots \ldots \ldots \ldots \ldots \ldots . \ldots 22.00$

$9-11$ (125 in a case) . . . . . . . . . . . . 22.00

\section{LILIUM AURATUM, Special strain.}

We have only about 20 cases of $8-9$ to sell at. 35.00

\section{LILIUM SPECIOSUM MAGNIFICUM MELPOMENE RUBRUM}

8-9 (200 in a case) . . . . . . . . . . . . 32.00

$9-11$ (140 in a case) ............... 35.00

COMBINATION CASES containing:

20 Album .....

50 Auratum.... $\} \ldots \ldots \ldots \ldots \ldots \ldots \ldots . \ldots \ldots . \ldots \ldots$

100 Magnificum.

\section{LILIUM ERABU}

$9-10(200$ in a case $) \ldots \ldots \ldots \ldots \ldots \ldots \ldots . \ldots .29 .00$

\section{LILIUM SINENSIS}

$6-8$ (400 in a case) . . . . . . . . . . . . 33.00

\section{LILIUM GIGANTEUM}

$6-8$ (400 in a case) . . . . . . . . . . . . . 34.00

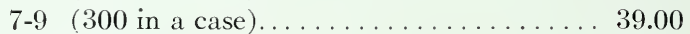

$8-10(225$ in a case $) \ldots \ldots \ldots \ldots \ldots \ldots \ldots . \ldots 45.00$

$9-10(200$ in a case $) \ldots \ldots \ldots \ldots \ldots \ldots . . . \ldots 48.00$

\section{LILIUM NOBELI}

$7-9(300$ in a case $) \ldots \ldots \ldots \ldots \ldots \ldots . \ldots . \ldots . \ldots 30 . . \ldots 0$

\section{VALLEY PIPS}

Per case

250 in a case..... . . . . . . . . . . . . . \$12.00

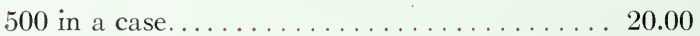

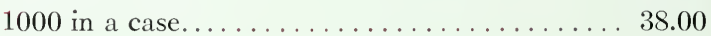

For new crop Lily Bulbs, see page 8

\section{ANEMONES}

A valuable cut flower for greenhouse growing, giving a succession of cut flowers. Grown in 55 to 60 degrees temperature.

De Caen. Single petals; fine colors. $\quad 100 \quad 1000$

1 year old bulbs (fine flowering size).. . \$2.75 $\$ 22.50$

2 year old bulbs (Iarge)... . . . . . . . $3.75 \quad 32.50$

St. Brigid. Double flowering, a wonderfuI strain and wide range of colors.

1 year old bulbs (fine flowering size).. . $4.00 \quad 35.00$

2 year old bulbs (large)............ $5.00 \quad 40.00$

His Excellency. Fire red. . . . . . . . . . . $3.50 \quad 27.50$ 


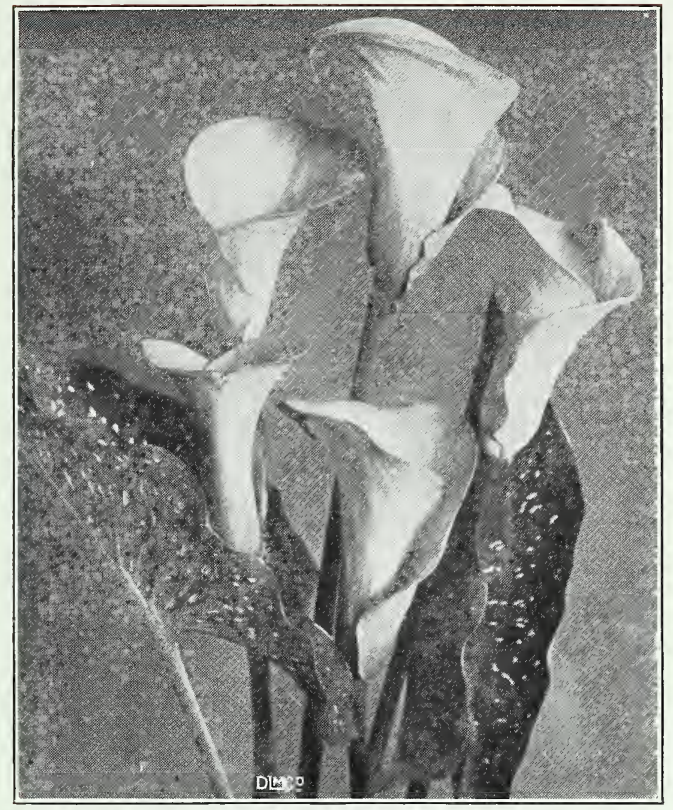

CALLA elliottiana

\section{CALLA BULBS}

(July Delivery)

\section{AETHIOPICA}

$100 \quad 1000$

$11 / 4-1 \frac{1}{2}$ inch. $\$ 8.50 \quad \$ 75.00$

$1 \frac{1}{2}-1 \frac{3}{4}$ inch. $16.00 \quad 150.00$

$13 / 4-2$ inch $19.00 \quad 185.00$

2 inch and up 27.50

\section{GODFREY}

$1-1 \frac{1}{4}$ inch $10.00 \quad 90.00$

$11 / 4-1 \frac{1}{2}$ inch. $17.50 \quad 165.00$

$1 \frac{1}{2}-1 \frac{3}{4}$ inch. 25.00

\section{FREESIAS}

NEW FREESIA

"SUPERFLORA" (Improved Purity)

Much superior to Purity. Will replace Purity in future.

$1 / 2$ inch..................\$18.00 per 1000

$5 / 8$ inch.................. 25.00 per 1000

(Continued on next page) 


\section{FREESIAS - continued}

PURITY (White)

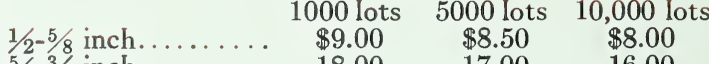

$5 / 8=3 / 4$ inch........... $18.00 \quad 17.00 \quad 16.00$

$3 / 4$ in $\mathrm{ch}$ and $\mathrm{up}$

(limited amount). 25.00

NOVELTIES Per 1000

Gold Cup. Yellow. . . . . . . . . . . . . . . . \$45.00

Lila. Light Iavender. . . . . . . . . . . . . . . . . . 47.50

Melba. Dark pink................. 45.00

White Star. White................. 42.50

COLORED FREESIAS

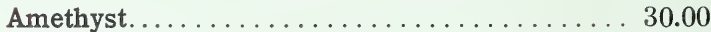

Aurora. Yellow . . . . . . . . . . . . . . . . 25.00

California. Yellow.................. 28.00

Carmencita. Light orange salmon. . . . . . . . . 25.00

Carrie Budau. Lavender pink. . . . . . . . . . . 32.00

General Pershing. Lavender. . . . . . . . . . . . . . 20.00

Giant Lavender........................ . 40.00

Golden Daffodil... . . . . . . . . . . . . . . . 55.00

Golden Treasure. . . . . . . . . . . . . . . . . . . . . . 55.00

Ilena. Rose Iavender................. . . 30.00

June Michelsen. Rose pink............. 40.00

Olivette. Carmine red.................... . 32.00

Splendens. . . . . . . . . . . . . . . . . . . . . 30.00

Yellow Prince.......................... 25.00

Mixed............................ 25.00

Above are $1 / 2$ inch bulbs

$5 / 8$ inch bulbs are $\$ 3.00$ per 1000 more and

$3 / 4$ inch bulbs are $\$ 7.00$ per 1000 more

\section{IRIS BULBS}

\section{TOP SIZE. Fall Delivery}

DUTCH IRIS Per 1000

Albert Cuyp. Yellow.................\$45.00

Anton Mauve. Blue........................ 40.00

David Bless. Blue, earlier than Imperator. . . . 55.00

Frans Hals. Yellow. . . . . . . . . . . . . . . . . . 45.00

Hart Nibbrig. Blue..................... . 45.00

Huchtenberg. Yellow..... . . . . . . . . . 45.00

Imperator. Blue . . . . . . . . . . . . . . . . . . 45.00

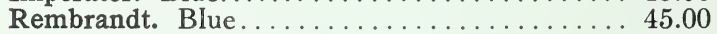

Van Everdingen. Yellow............... 45.00

Yellow Queen. Yellow................... 60.00

E. B. Garnier. Blue. Considered better than

David Bless........................ 55.00

Van Der Helst. Yellow................... 55.00

\section{SPANISH IRIS}

Alex von Humboldt. Blue . . . . . . . . . . . . . 35.00

Belle Chinoise. Yellow. . . . . . . . . . . . . . 40.00

British Queen. White. . . . . . . . . . . . 35.00

Cajanus. Yellow....................... 40.00

Chrysolora. Yellow.................. 35.00

Darling. Blue............ . . . . . . . . . . 35.00

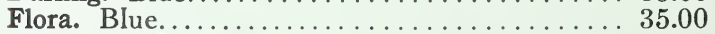

King of the Blues. Blue.............. 35.00

La Reconnaissance. Bronze................. 35.00

Louise. Lavender. . . . . . . . . . . . . . . . . . . . 35.00

Queen Wilhelmina. White............. 35.00

Mixed colors ........................ 35.00

\section{OTHER IRIS}

Wedgewood. Giant blue............ $\$ 20.00$ per 100

First size bulbs, 10 per cent lower than above top size. 
Named kinds, yellow, red, mauve, primrose and

white with eye.................\$17.50

Mixed....................... 15.00

\section{RANUNCULUS}

Selected Hybrids

Per 1000

1 year old, No. 1. . . . . . . . . . . . . . . . \$22.00

2 year old, No. 1.................... 30.00

\section{Fancy Varieties}

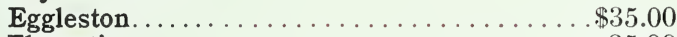

Florentine. . . . . . . . . . . . . . . . . . 35.00

Palestine....................... . 35.00

\section{CROCUS}

Purpurea grandiflora (Extra purple) Per 1000

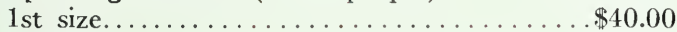

2nd size........................... 380

Mammoth Yellow

1st size......................... 45.00

2nd size........................ 38.00

Named varieties in white, blue, purple and striped.

1 st size. . . . . . . . . . . . . . . . . 28.00

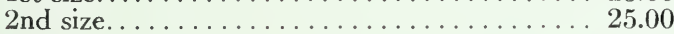

\section{HYACINTHS}

Miniature size, $12-15 \mathrm{ctm} \ldots \ldots \ldots \ldots \ldots \$ 7.00 \quad \$ 60.00$

Bedding size, $15-16 \mathrm{ctm} \ldots \ldots \ldots \ldots \ldots 8.50 \quad 77.50$

Third size, $16-17 \mathrm{ctm} \ldots \ldots \ldots \ldots \ldots \ldots . \ldots 10.00 \quad 90.00$

Second size, $17-18 \mathrm{ctm} \ldots \ldots \ldots \ldots \ldots . \ldots 11.00 \quad 97.50$

First size, $18-19 \mathrm{ctm} \ldots \ldots \ldots \ldots \ldots \ldots .12 .50 \quad 110.00$

Exhibition size, $19 \mathrm{ctm}$. . . . . . . . . $15.00 \quad 125.00$

All above sizes are supplied in all of the following colors:

\section{WHITE AND BLUSH}

L'Innocence. Pure white, excellent forcer; most popular.

La Grandesse. Pure white; for early forcing.

\section{YELLOW}

$\begin{gathered}\text { City of Haarlem } \\ \text { Yellow Hammer }\end{gathered}$
ROSE, PINK AND RED
$\begin{gathered}\text { Gertrude } \\ \text { Gigantea }\end{gathered}$
$\begin{gathered}\text { Ling of the Belgians) } \\ \text { La Victoire } \\ \text { Quarconi }\end{gathered}$
Queen of the Pinks
BLUE AND PURPLE
$\begin{array}{cc}\text { Bismarck } & \text { King of the Blues } \\ \text { Grand Maitre } & \text { Queen of the Blues }\end{array}$

\section{GRAPE-HYACINTHS}

$\begin{array}{lccc}\text { Large size } & \text { 1st size } & \text { 2nd size } \\ \text { Dark Blue........ } & \$ 18.00 & \$ 15.00 & \$ 12.00 \\ \text { Heavenly Blue .... } & 22.00 & 17.50 & 14.00\end{array}$




\section{NARCISSI}

\section{AMERICAN GROWN}

LARGE YELLOW TRUMPETS Per 1000

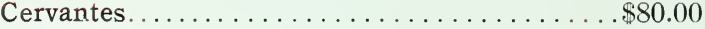

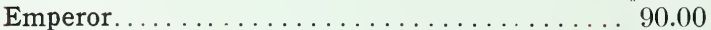

Golden Spur........................ 90.00

King Alfred............. . . . . . . . 140.00

Minister Talma. . . . . . . . . . . . . . . . . . 105.00

Princeps. . . . . . . . . . . . . . . . . . . . 75.00

Tresserve............... . . . . . . . . . 150.00

Trumpet Major................... 90.00

LARGE WHITE AND

SULPHUR WHITE TRUMPETS

Madame De Graaff......................100.00

\section{LARGE BI-COLORS}

Empress........................... . 90.00

Glory of Sassenheim. . . . . . . . . . . . . 100.00

Spring Glory........ . . . . . . . . . . . . . . 105.00

Victoria...................... 85.00

\section{BARRI}

Conspicuus.

Firebrand. .

Masterpiece

\section{INCOMPARABILIS}

Lucifer

Sir Watkin

\section{JONQUILS}

Single.

Double................. . . . . . . . . . 55.00

Campernelle Orange Queen............... 75.00

Campernelle Regulosus Single............ 55.00

Campernelle Regulosus Double............ 65.00

\section{LEEDSI}

White Lady...................... 70.00

White Queen..................... 90.00

\section{POETAZ}

Aspacia.

Elvira.

Lawrence Koster.

Orange Cup

Ornatus.

Recurvus (Pheasant's-eye).................. 50.00

\section{POETICUS}

POLYANTHUS

Grand Soliel d'Or................. 85.00

\section{DOUBLE NARCISSI}

Albo Pleno Odorato.................. 80.00

Double Incomparable.... . . . . . . . . . . . . . 75.00

Orange Phoenix........................ 115.00

Sulphur Phoenix.................... 95.00

Von Sion....................... 95.00

\section{MIXTURES OF NARCISSI}

All kinds........................... . 50.00 Extra Selected...................... 65.00

\section{PAPERWIITES Per 1000}

$12 \mathrm{ctm} .(1250$ in a case $) \ldots \ldots \ldots \ldots \ldots \ldots . \ldots . \ldots 33.00$

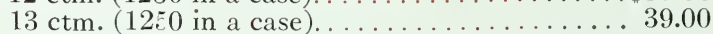

$14 \mathrm{ctm} .(1000$ in a case)............. 45.00

$100 \quad 1000$

GRAND SOLEIL D'OR ........... \$11.00\$100.00 CHINESE SACRED LILIES . . . . . . $9.00 \quad 85.00$ 


\section{TULIPS}

DARWIN TULIPS Per 1000

Baron de la Tonnaye. Rose. . . . . . . . . . . . . . . . \$28.00

Bartigon. Red. ....................... 29.00

Clara Butt. Salmon rose... . . . . . . . . . . . . . . 23.00

Dream. Lilac. ... . . . . . . . . . . . . . . . . . . 38.00

Farncombe Sanders. Scarlet.............. 26.00

La Candeur (White Queen) ............... 30.00

La Tulipe Noire. Black.................. 40.00

La Notre. Rose.... . . . . . . . . . . . . . . . 32.00

Mme. Krelage. Rose. . . . . . . . . . . . . . . . . . 27.00

Massachusetts. Rose... . . . . . . . . . . . . 23.00

Pride of Haarlem. Rose........ . . . . . 27.00

Princess Elizabeth. Lilac rose............... 30.00

Prof. Rauwenhoff. Red................... 30.00

Rev. Ewbank. Lilac. . . . . . . . . . . . . . . 32.00

Roi d'Islande. Violet rose . . . . . . . . . . . . . 49.00

William Copland. First size, Iavender........ 29.00

William Copland. Large size, Iavender. . . . . . . . 32.00

William Pitt. Scarlet. . . . . . . . . . . . . . . . . 30.00

Darwin Tulips Mixed.................. 25.00

\section{SINGLE EARLY TULIPS}

Artus. Scarlet. . . . . . . . . . . . . . . . . . . . . 23.00

Belle Alliance. Scarlet. . . . . . . . . . . . . . . . 32.00

Brilliant Star. Scarlet. . . . . . . . . . . . . . . 32.00

Couleur Carc̈inal. Scarlet. . . . . . . . . . . . . 33.00

Cramoisi Brillant. Scarlet. . . . . . . . . . . . . . . 21.00

Flamingo. Scarlet. . . . . . . . . . . . . . . . . . . 32.00

Fred Moore. Orange................. 29.00

Ibis. Pink ................. . . . . . . . . . 39.00

Keizerskroon. Red and yellow........... 50.00

La Reine Maxima. White. . . . . . . . . . . . . 29.00

Mon Tresor. Yellow . . . . . . . . . . . . . . . . . 57.00

Prince of Austria. Orange red............... . 29.00

Prosperity. Rose. . . . . . . . . . . . . . . . . . . . 32.00

Rising Sun. Yellow. . . . . . . . . . . . . . . 39.00

Rose Gris de Lin. Rose. . . . . . . . . . . . . . . 27.00

Thomas Moore. Bronze. . . . . . . . . . . . . . . 29.00

Vermillon Brillant. Scarlet. . . . . . . . . . . . 38.00

Yellow Prince....................... 35.00

\section{DOUBLE EARLY TULIPS}

Couronne d'Or. Yellow. . . . . . . . . . . . . . . . . 39.00

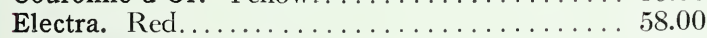

E1 Toreador. Scarlet. . . . . . . . . . . . . . . . 64.00

Imperator Rubrorum. Scarlet............. 39.00

Mr. Van Der Hoeff. Yellow. . . . . . . . . . . . . . 39.00

Murillo. White..................... 25.00

Peach Blossom. Pink................. 38.00

Rubra Maxima. Crimson. . . . . . . . . . . . . . . . 35.00

Schoonoord. White.................. 34.00

Tearose. Yellow. . . . . . . . . . . . . . . . . . 29.00

Tournesol. Red and yellow................ 39.00

Vuurbaak. Orange scarlet. . . . . . . . . . . . . 38.00

Double Mixed ......................... 27.00

\section{COTTAGE TULIPS}

Gesneriana Lutea. Yellow. . . . . . . . . . . . . . . 2 27.00

Gesneriana Spathulata. Crimson. . . . . . . . . 29.00

Inglescombe Pink . . . . . . . . . . . . . . 27.00

Inglescombe Yellow. . . . . . . . . . . . 23.00

Inglescombe Scarlet. . . . . . . . . . . . . . . . 29.00

Moonlight. Yellow. . . . . . . . . . . . . . . . . . 40.00

Mrs. Moon. Yellow. ....................... 47.00

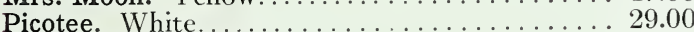

Mayflowering Mixed ............. 23.00 


\section{New Crop LILY BULBS}

\section{LILIUM REGALE}

(Ready Fall)

Per 100

4-5 inch. $\$ 15.00$

5-6 inch 20.00

6-8 inch 27.50

7-9 inch. 45.00

\section{LILIUM CANDIDUM}

(Delivery July, 1930, on arrival)

$100 \quad 1000$

$18-20 \mathrm{ctm}$. , packed 500 to a case. . . . $\$ 11.00 \$ \$ 85.00$

20-22 ctm., packed 400 to a case..... $12.50 \quad 100.00$

$22-34 \mathrm{ctm}$., packed 300 to a case...... $14.00 \quad 115.00$

$24-20 \mathrm{ctm}$., packed 250 to a case. . . . $16.00 \quad 130.00$

$26 \mathrm{ctm}$. up packed 200 to a case...... $17.50 \quad 145.00$

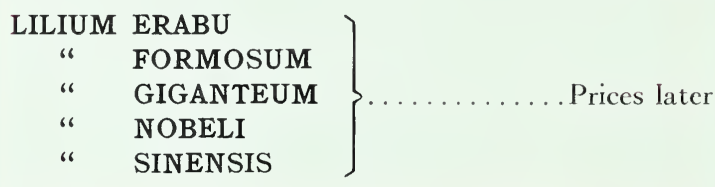

\section{ALL PRICES ARE SUBJECT TO CHANGE WITHOUT NOTICE}

WE do not warrant in any way, express or implied, the contents or the description, purity, productiveness or any other matter of any seeds, bulbs, or plants sold by us, and we will not be in any way responsible for the crop. If the purchaser does not accept these goods on above tcrms, no sale is made thercof, and he must return them at once, and money will bc refunded. Subject to the above conditions, we makc this sale at the modcrate prices at which we sell our goods.

All claims must be made within five days after receipt of goods.

\section{2e \\ STANDARD BULB CO. INC. 105 West 28th Street New York, N. Y.}

Phone: LACkawanna 5790 